\title{
Obesidade e gestação: a importância da correlação na avaliação dos riscos materno-fetais
}

\author{
Obesity and pregnancy: the importance of correlation in the evaluation of maternal and fetal risks \\ Obesidad y embarazo: la importancia de la correlación en la evaluación del riesgo materno-fetal
}

Paula Zamboti Brandão, Thauane Barbosa da Silva², Emílio Conceição de Siqueira ${ }^{3}$

Como citar esse artigo. Brandão, PZ; da

Silva, TB; de Siqueira, EC. Obesidade e gestação: a importância da correlação na avaliação dos riscos materno-fetais. Revista Pró-UniverSUS. 2019 Jul./Dez.; 10 (2): $18-23$

\section{Resumo}

A obesidade define-se por excesso de tecido adiposo na composição corporal de um indivíduo, a mesma possui causa multifatorial e se caracteriza por altas taxas de agravos e mortes no mundo Em relação a gestação, complicações como a diabetes, pré-eclâmpsia, eclampsia e parto cirúrgico são algumas das intercorrências que podem afetar a gestante; enquanto o bebê pode apresentar índices elevados de malformação fetal, macrossomia e hipoglicemia neonatal.O presente trabalho constitui-se de uma pesquisa exploratória que usa como procedimento o levantamento bibliográfico e a coleta de dados de artigos científicos das áreas examinadas. A nutrição da mulher influencia tanto o período gestacional quanto o puerpério e está diretamente ligada a possibilidade de intercorrências na saúde da mãe e do concepto. A gestante está sujeita a complicações como diabetes, desordens hipertensivas e parto cesariana enquanto o recém nato pode apresentar problemas decorrentes de malformação fetal, peso elevado para a idade gestacional além de quadros de hipoglicemia neonatal. Durante a gravidez, o organismo feminino sofre diversas alterações a fim de proporcionar o desenvolvimento do embrião. O peso da mulher antes da gestação tem estrita relação com o aumento ponderal durante a gravidez, além de influenciar de forma direta na saúde da mãe e do concepto. Assim, a obesidade associa-se de maneira direta ao prognóstico da gestação. Desta maneira, a perda de peso, prática regular de atividades físicas e alimentação saudável devem ser estimuladas pelos profissionais de saúde, com o fim de promover a saúde materna e fetal.

Palavras-chave: Obesidade, Gestação, Feto.

\begin{abstract}
Obesity is defined by excess fat in the body composition of a person, it has multifactorial causes and is characterized by high rates of injuries and deaths in the world. In relation to pregnancy complications such as diabetes, preeclampsia, eclampsia and surgical birth are some of the complications that can affect pregnant women; while the baby may have high rates of fetal malformations, macrosomia and neonatal hypoglycemia. This study consists of an exploratory research using the procedure of the literature review and data collection of scientific articles of the areas examined. Woman nutrition influences both the pregnancy and the postpartum period and is directly linked to the possibility of complications in the health of the mother and fetus. The patient is subject to complications such as diabetes, hypertensive disorders and cesarean delivery while the newly born can present problems due to fetal malformation, high weight for gestational age as well as episodes of hypoglycemia neonatal. During pregnancy, the female body undergoes several changes in order to provide the developing embryo. The weight of the woman before pregnancy has close relationship with the weight gain during pregnancy, and influence directly on the health of the mother and fetus. Thus, obesity is associated with a direct way to pregnancy outcome. In this way, weight loss, regular physical activity and healthy eating should be encouraged by health professionals, in order to promote maternal and fetal health.
\end{abstract}

Keywords: Obesity, Gestation, Fetus.

\footnotetext{
Afiliação dos autores:

1. Universidadede Vassouras, Vassouras, RJ, Brasil. Email: paulazamboti@hotmail.com ORCID: https://orcid.org/0000-0003-3260-3036

2. Universidadede Vassouras, Vassouras, RJ, Brasil. Email: thauane.thata@hotmail.com ORCID: https://orcid.org/0000-0003-0996-3519
}

3. Universidadede Vassouras, Vassouras, RJ, Brasil. Emailemiliouba@uol.com.br ORCID:Orcid: https://orcid.org/0000-0002-8489-6531 


\section{Resumen}

La obesidad se define como el exceso de grasa en la composición corporal de un individuo, tiene una causa multifactorial y se caracteriza por altas tasas de lesiones y muertes en todo el mundo. Con respecto al embarazo, complicaciones como diabetes, preeclampsia, eclampsia y el parto quirúrgico son algunas de las complicaciones que pueden afectar a la mujer embarazada; mientras que el bebé puede presentar altas tasas de malformación fetal, macrosomía e hipoglucemia neonatal. Este estudio es una investigación exploratoria que utiliza como procedimiento la encuesta bibliográfica y la recopilación de datos de artículos científicos de las áreas examinadas. La nutrición de las mujeres influye tanto en el período gestacional como en el posparto y está directamente relacionada con la posibilidad de complicaciones en la salud maternoinfantil. La mujer embarazada está sujeta a complicaciones como diabetes, trastornos hipertensivos y parto por cesárea, mientras que el recién nacido puede tener problemas como resultado de malformación fetal, alto peso para la edad gestacional e hipoglucemia neonatal. Durante el embarazo, el organismo femenino sufre varios cambios para promover el desarrollo del embrión. El peso de las mujeres antes del embarazo está estrechamente relacionado con el aumento de peso durante el embarazo e influye directamente en la salud de la madre y el concepto. Por lo tanto, la obesidad está directamente asociada con el pronóstico del embarazo. Por lo tanto, los profesionales de la salud deben alentar la pérdida de peso, la actividad física regular y una alimentación saludable para promover la salud materna y fetal.

Palabras clave: Obesidad, Gestación, Feto.

\section{Introdução}

A obesidade define-se por excesso de tecido adiposo na composição corporal de um indivíduo ${ }^{1}$, a mesma possui causa multifatorial e se caracteriza por altas taxas de agravos e mortes no mundo ${ }^{2}$. De acordo com o Ministério da Saúde 1 em cada 5 pessoas apresenta sobrepeso, tendo o índice da obesidade crescido cerca de $7 \%$ entre 2006 e $2016^{3}$.

Este trabalho tem o intuito de reunir dados acerca da relação entre o índice ponderal elevado e a gestação. Visa alertar a respeito das complicações nos períodos pré e pós termos e enfatizar os riscos para a progenitora e o concepto.

Um dos parâmetros aceitos mundialmente para o diagnóstico de obesidade consiste na relação entre massa corpórea e estatura definida como índice de massa corporal (IMC). No entanto é um critério subjetivo pois não em leva em conta a relação tecido adiposo e massa muscular ${ }^{4}$.

Outrossim, é importante destacar a relação direta entre e obesidade e outras comorbidades como dislipidemias, doenças cardiovasculares, diabetes mellitus tipo II dentre outras patologias que favorecem a redução da expectativa de vida ${ }^{5}$.

Em relação à gestação, complicações como a diabetes, pré-eclâmpsia, eclampsia e cesariana são algumas das intercorrências que podem afetar a gestante; enquanto o bebê pode apresentar índices elevados de malformação fetal, macrossomia e hipoglicemia neonatal ${ }^{2}$.

Em liame à gestação, o excesso de tecido adiposo expande sua magnitude, pois a incidência de intercorrências pré e pós-natais é ampliada. Segundo a Organização Mundial da Saúde ${ }^{7}$, o controle do ganho de massa durante a prenhez é uma rotina de baixo custo e de alta valia para o estabelecimento de medidas que visem à míngua dos riscos maternos e fetais, contribuindo para a promoção da saúde de ambos os envolvidos, na tentativa de oportunizar melhores condições para o parto e vida pós-natal ${ }^{2}$.

\section{Material e Método}

Este artigo visa a construção de uma pesquisa exploratória que utiliza como coleta de dados o levantamento bibliográfico de artigos científicos e livros da área examinada.

\section{Resultados}

A nutrição da mulher influencia tanto o período gestacional quanto o puerpério está diretamente ligada a possibilidade de intercorrências na saúde da mãe e do concepto. A alimentação inadequada neste período pode se associar a alterações na expressão genética e aumentar a suscetibilidade as intercorrências, inclusive durante o parto e puerpério ${ }^{8}$.

Durante a gravidez, o organismo feminino sofre diversas alterações a fim de proporcionar o desenvolvimento do embrião. Desta maneira o acréscimo ponderal nessa ocasiãose enquadra como fisiológico, sendofundamental para o crescimento do feto 9. Não obstante, o ganho de peso deve levar em consideração os valores ponderaispré gestacionais e seguir recomendações que limitam o aumento de massa por trimestre. É recomendado que mulheres com estado nutricional pré-gravídico de baixo peso ganhem de 12,5 a $18 \mathrm{~kg}$ durante a gestação, enquanto as obesas devem se limitar ao ganho de 5 a $9 \mathrm{~kg}$ até o fim da prenhez. No entanto, mais de $65 \%$ das gestantes excedem os valores recomendados o que contribui para a incidência de intercorrencias ${ }^{10}$.

Vale destacar, que devido a gestação, a mulher necessita de um acréscimo das reservas energéticas para suprir o aumento do metabolismo basal, desta maneira faz-se necessário maior ingesta calórica durante este período. A alimentação deve seguir os mesmos critérios qualitativos de uma adulta não prenha, baseando-se no consumo de cereais, leguminosas, vegetais crus, frutas e produtos de origem animal, evitando o consumo de álcool e alimentos industrializados, bem como o excesso 
de açúcar e sal ${ }^{11}$.

O peso da mulher antes da gestação tem estrita relação com o aumento ponderal durante a gravidez, além de influenciar de forma direta na saúde da mãe e do concepto. Estudos demonstram que o sobrepeso e a obesidade anterior ao período da gravidez predispõem às diversas intercorrências, dentre elas destaca-se a préeclâmpsia, que aparenta ser mediada pela proteína $\mathrm{C}$ reativa e triglicerídeos, porém ainda são desconhecidos os mecanismos fisiopatológicos desta relação ${ }^{8}$.

É recomendado que as mulheres com excesso de peso recebam orientações nutricionais desde o período pré-gestacional até o período pós-gestacional, com intuito de reduzir as complicações perinatais. Assim, a alimentação saudável, a prática regular de atividades físicas e a normalização do peso são fatores que devem ser estimulados não só durante a gravidez, mas de forma de permanente.

Contudo, apesar de a recomendação de ganho de peso seja mais restrito para as mulheres obesas ou com sobrepeso, esteé o grupo que mais apresenta inadequação do ganho de peso. Estudos mostram que mulheres com sobrepeso no início da prenhez, além de aumentarem o peso excessivamente durante o período gestacional, tendem a não recuperar o peso pré-gestacional após o parto $^{6}$.

É sabido que a gestação por si só, se caracteriza como um período crítico que traz consigo o risco gravídico necessitando desta maneira de assistência à saúde ampliada, o que exige maior atenção por partes dos profissionais. Quando a obesidade está aliada a gravidez a preocupação é redobrada pois as possíveis complicações para a mãe e o feto são exacerbadas, caracterizando por vezes essa gestação como de alto risco.

\section{Diabetes gestacional e diabetes tipo 2}

Estima-se que cerca de 415 milhões de pessoas apresentam Diabetes Mellitus (DM) e cerca de 318 milhões têm intolerância à glicose no mundo, sendo o Brasil o quarto país com maiores taxas de diabetes. Dentre a população feminina, sabe-se que a o Diabetes Mellitus Gestacional (DMG) é o principal fator de risco para o desenvolvimento de diabetes mellitus tipo 2 e síndrome metabólica. Assim, a DMG durante a gravidez apresenta-se como um importante problema na atualidade, tanto pelo risco de intercorrências maternas e fetais quanto pelo aumento da sua prevalência ${ }^{12}$.

A mulher, durante a gestação, pode apresentar diabetes mellitus gestacional ou ter diabetes mellitus diagnosticada neste período. A DMG se caracteriza pela hiperglicemia detectada pela primeira vez durante a gestação, porém com níveis glicêmicos sanguíneos que não alcançam os critérios diagnósticos para DM. Já a DM diagnosticada na gestação ocorre quando a mulher, sem diagnóstico prévio da doença, apresenta hiperglicemia compatíveis com os critérios de diagnósticos da OMS para DM na ausência de gravidez ${ }^{12}$.

Durante a prenhez, a mulher apresenta um estado de resistência insulínica. Este ocorre pela baixa atuação da insulina em seus receptores, devido a ação de hormônios produzidos na placenta ou hormônios maternos aumentados durante o período gestacional, tais como o cortisol e o lactogênio placentário. Como consequência, em mulheres saudáveis, ocorre o incremento da produção de insulina para compensar a resistência ao hormônio que ocorre na gestação. Contudo, esse mecanismo não ocorre em gestantes que já estão com sua capacidade de produção de insulina no limite. Dessa forma, não será possível incrementar a produção deste hormônio, como f, o que pode levar à diabetes nesse período ${ }^{12}$.

$\mathrm{O}$ risco de intercorrências à mãe e ao filho são diretamente proporcionais ao aumento da glicemia materna. As principais complicações da DMG são para a mãe a cesariana e a pré-eclâmpsia, já ao concepto são prematuridade, macrossomia, hipoglicemia, distocia de ombro e morte perinatal ${ }^{13}$.

Sendo assim, o tratamento do DMG reduz a morbidade perinatal e favorece à qualidade de vida materna. O tratamento muito se assemelha ao que recomendado em outras formas de diabetes, ele inclui a prática de atividades físicas, dieta individualizada e uso de medicações, sendo a insulinoterapia a melhor alternativa ${ }^{13}$.

A presença de DMG associada a obesidade apresenta efeito deletério maior do que cada um dos fatores isoladamente, inclusive sobre a ocorrência de malformações congênitas e macrossomia.

\section{Desordens hipertensivas}

Segundo o ministério da Saúde (MS) a hipertensão arterial sistêmica (HAS) é caracterizada como a elevação da pressão arterial com valores superiores à $140 \mathrm{mmhg}$ de pressão sistólica e 90 mmhg de componente diastólico ${ }^{14}$. Em comparação com hipertensão que antecede a gestação, a alteração pressórica que se manifesta durante o período gravídico pode surgir como resultado de uma má adaptação do corpo às modificações gestacionais, evidenciando um quadro de maior apresentação clínica. Essa alteração hemodinâmica é apontada pelo MS como responsável por mais de $30 \%$ dos óbitos maternos no Brasil, sendo uma das maiores causas de morte em gestantes.

Existem diferentes classificações para a HAS na gestação de acordo com as características apresentadas e tempo de aparecimento. Quando o diagnóstico ocorre anterior a gravidez ou antes da $20^{\circ}$ semana é posto 
como uma hipertensão crônica. No entanto, se o rastreio é feito posterior a este período, por volta do $5^{\circ}$ mês de gestação $\left(20^{\circ}\right.$ semana $)$ é classificado como hipertensão gestacional. Pode ainda ocorrer concomitante à essa alteração a presença de proteinúria $(\geq 0,3 \mathrm{~g}$ de proteína em urina de 24 horas) sendo classificado em préeclâmpsia (PE), sendo essa decorrente da hipertensão gestacional ou sobreposta à hipertensão crônica após a $20^{\circ}$ semana $^{15}$. Outrossim, a PE pode aparecer antes da $20^{\circ}$ semana em casos onde existe em concomitância a presença de Neoplasia Trofoblástica Gestacional ${ }^{16}$.

A PE é um agravo de etiologia desconhecida e sua fisiopatologia não é bem esclarecida, mas acreditase que o organismo da gestante com essa patologia curse com alterações morfofuncionais, como à não invasão de células trofoblasticas ou invasão deficiente determinando a existência de vasos de alta resistência e baixa circulação placentária. Como resultado, ocorre espasmo arterial com consequente diminuição do calibre dos vasos e má perfusão sanguínea, o que corrobora para o aumento da pressão arterial, hipóxia placentária e lesão endotelial com alteração da agregação plaquetária ${ }^{15}$.

Por ser uma doença de causa multifatorial, diversos fatores influenciam no seu aparecimento como gravidez de mulheres primigestas, extremos de idade da gestante, diabetes, neuropatias, história familiar prévia da doença, neoplasia trofoblastica e a obesidade aliada à gestação ${ }^{16}$. O peso da mãe é um fator de risco independente para o surgimento da doença. Estudos mostram que pejadas com índice de massa corporal maior que $30 \mathrm{~kg} / \mathrm{m}^{2}$ aumentam em duas a três vezes a probabilidade de cursar com PE, sendo que o risco pode dobrar dependendo da faixa de ganho de peso anterior à gestação ${ }^{6}$.

Acredita-se que existam dois diferentes mecanismos em que a obesidade seria o fator desencadeante da PE. A explicação para a primeira teoria seria a presença de adipócitos hipertrofiados em mulheres obesas que ampliariam a resposta inflamatória no organismo. Com esse mecanismo exacerbado, a liberação de seus mediadores também sofreria elevação, como a proteína c reativa, e estes poderiam alterar as funções das células endoteliais promovendo a PE. O outro mecanismo tem explicação baseada na função hepática, que com o aumento das células gordurosas, permitiria o aumento de ácidos graxos livres e espécies reativas de oxigênio além de promover a redução de agentes antioxidantes. Essas modificações levariam ao estresse oxidativo inclusive a nível placentário ocasionando a disfunção endotelial e posterior $\mathrm{PE}^{17}$.

\section{Tromboembolismo}

O tromboembolismo venoso (TEV) pode ser definido como a combinação de duas patologias: a embolia pulmonar e da trombose venosa profunda. A primeira se caracteriza pela obstrução por coágulos nos vasos pulmonares enquanto a segunda ocorre nas veias profundas dos membros inferiores. A tríade de Virchow explica que a lesão endotelial, estase venosa e alterações da constituição do sangue que levam ao estado de hipercoagulabilidade são determinantes para a ocorrência do $\mathrm{TEV}^{18}$.

Os componentes dessa tríade ocorrem fisiologicamente no curso da gravidez. Alterações no corpo da grávida levam ao aumento da capacidade e distensibilidade dos vasos, o que por si só pode aumentar a estase sanguínea. Além disto os níveis de fatores da coagulação como II, VII, VIII, X e de von Willebrand estão elevados durante a gravidez juntamente com a produção de fibrinogênio o que contribui ainda mais para o estado pró-trombótico encontrado nas gestantes. Esses eventos tromboembólicos estão entre as principais causas de morbidade materna e mortalidade no período gestacional e puerperal e ampliam as chances de ocorrência quando aliados a fatores de risco como a obesidade. Esta última tambémexacerba as chances de aparecimento de outras doenças como hipertensão, diabetes do tipo 2 e hipercolesterolemia que somadas ampliam as chances de insuficiência venosa e estase sanguínea ${ }^{6}$.

\section{Abortamento}

O abortamento espontâneo se caracteriza pela descontinuação involuntária da gestação até a $22^{\circ}$ semana ou feto com peso inferior a 500 gramas, que pode cursar ou não com a expulsão do concepto ${ }^{19}$. Ainda não existem comprovações cientificas da relação direta entre o aborto e a obesidade na gravidez (artigo obesidade e infertilidade), mas estudos demonstram que a taxa de abortamento entre mulheres obesas é superior àquelas que não possuem tal morbidade ${ }^{6}$. Uma das teorias que tentam explicar tal associação, é que devido à grande quantidade de ácidos graxos livres no fluido folicular existe o comprometimento da qualidade dos ovócitos dessas mulheres ${ }^{20}$.

\section{Macrossomia}

A macrossomia é a intercorrência mais comumente observada em gestações complicadas por hiperglicemia diária ou diabetes. Macrossomia define-se como peso ao nascimento maior que $4000 \mathrm{~g}$, independente de variações demográficas e do tempo gestacional. Além disso, pode-se relacionar o peso fetal de acordo com a idade gestacional, que caracterizará os neonatos grandes para a idade gestacional (GIG) quando o peso ultrapassar o percentil $90^{21}$.

$\mathrm{O}$ aumento do IMC relaciona-se com a 
macrossomia na medida em que a ocorrência de resistência insulínica materna leva a hiperinsulinemia fetal; visto que a insulina está diretamente relacionada ao crescimento do feto, o aumento deste hormônio é um importante fator para o crescimento intrauterino. Ademais, há maior aporte de ácidos graxos livres para o feto, pois em pacientes com resistência insulínica há lipases que clivam os triglicerídeos presentes em excesso. Outrossim, com as modificações ocorridas no ambiente fetal, a síntese e secreção da leptina é alterada, modificando o metabolismo dos adipócitos dos fetos $^{6}$. Devido a fisiopatologia descrita anteriormente, observa-se que mulheres obesas geram filhos GIG cerca de 1,2 a 1,8 vezes mais do que as que possuem IMC adequado $^{22}$.

A macrossomia leva a intercorrências tanto à mãe quanto ao feto, podendo deixar sequelas definitivas nos neonatos. Fetos GIG apresentam maior risco de morte intrauterina, paralisia facial e do plexo braquial, asfixia, aspiração de mecônio hipoglicemia, hiperbilirrubinemia neonatal, distocia de ombro, cardiomiopatia hipertrófica e uso de unidade de terapia intensiva. Ademais, os neonatos que apresentam macrossomia podem apresentar outras intercorrências ao longo da vida, como sequelas neurológicas, dislipidemia, obesidade, resistência insulínica e diabetes mellitus ${ }^{21}$.

Apesar de a gestação não complicada com suspeita de macrossomia não ser indicação de parto operatório, a macrossomia tem sido associada a ocorrência de cesáreas. A macrossomia também pode levar a complicações maternas, como trabalho de parto prolongado, parto operatório, laceração do trato urogenital, atonia e ruptura uterina ${ }^{23}$.

\section{Malformação fetal}

A obesidade materna também se relaciona a prevalência de anomalias congênitas. Dentre as anomalias observadas destacam-se as cardíacas, os defeitos do tubo neural, anencefalia e as anormalidades da parede abdominal. Estudos relacionam o aumento da ocorrência de defeitos de tubo neural a excessiva interferência de tecido adiposo no metabolismo dos folatos, com perda do efeito protetor do ácido fólico nessas gestantes ${ }^{6}$. O risco de malformações congênitas em mulheres obesas também pode se relacionar à dificuldade na visualização do ultrassom, durante o período pré-natal. Além disso, há prejuízo na realização da ecografia, a qual visualização do cordão umbilical, do coração e da medula é comprometida na quando o IMC é maior que cerca de $36 \mathrm{~kg} / \mathrm{m}^{2}$.

\section{Complicações fetais}

Estudos demonstram que a hiperglicemia durante a gestação aumenta o risco pós-natal de distúrbios metabólicos e pode afetar o desenvolvimento neurológico; a longo prazo associa-se à obesidade e diabetes mellitus. Além disso, neonatos de mães diabéticas podem apresentar hipoglicemia, devido a secreção aumentada de insulina permanecer após o parto.

\section{Discussão}

A gravidez é um estágio pelo qual o corpo da mulher sofre diversas modificações fisiológicas com o intuito de gerar um novo ser. Ela por si só aumenta os riscos de patologias endocrinometabólicas, como estados de hipercoagulabilidade e resistência insulínica. Dessa forma quando a mulher obesa engravida esses riscos somam-se aos relacionados à obesidade, ampliando a possibilidade de intercorrências para a mãe e o bebê.

Dessa forma,o pré-netal é importante para preservar o bem-estar materno e fetal, o que reduz os riscos de intercorrências e propicia o tratamento precoce caso ocorram. Sendo assim todas as gestantes devem realizar a avaliação periódica dos níveis de pressão arterial, glicemia, além de colesterol e triglicerídeos, já que os valores dos mesmos podem aumentar neste período.

O aconselhamento nutricional é de extrema importância neste período, visto que culturalmente na sociedade em que estamos inseridos têm-se a visão de que durante a gravidez a mulher deve aumentar a ingesta alimentar consumindo por ela e pelo feto além de que a escolha do alimento não segue os valores nutricionais e sim seus desejos com crenças que reproduzem este pensamento.

Neste contexto, o acompanhamento de uma equipe multiprofissional é de extrema importância para a evolução da gravidez. O apoio de nutricionistas, profissionais de educação física, fisioterapeutas, dentre outros especialistas da área da saúde ajudam a mudar conceitos preestabelecidos e direcionam a mulher a ter hábitos saudáveis e prorroga-los para a fase pósgestacional.

Outrossim, é indicado que a mulher obesa que tenha planos de gestar seja orientada a modificar seus hábitos antes de engravidar, sendo encorajada pela equipe de saúde a buscar uma reeducação alimentar ea prática de atividades físicas, com o intuito melhorar o índice ponderal e a qualidade da saúde visando um melhor prognóstico gestacional. Contudo, frequentemente isso não é possível, cabendo ao profissional orientar quanto aos riscos gestacionais e pós-gestacionais que a obesidade pode acarretar e atuando da melhor forma mesmo em tais casos para garantir a segurança gestacional. 


\section{Considerações Finais}

Assim, a obesidade associa-se de maneira direta ao prognóstico da gestação. Desta maneira, a perda de peso, prática regular de atividades físicas e alimentação saudável devem ser estimuladas pelos profissionais de saúde, com o fim de promover a saúde materna e fetal.

\section{Referências}

1. Sociedade Brasileira de Endocrinologia e Metabologia [homepage na internet]. Saiba como definir a obesidade [acesso em 30 jun 2019]. Disponível em: https://www.sbemps.org.br/315-saiba-como-definir-a-obesidade

2. Anjos LA. Diagnóstico de obesidade e determinação de requerimentos nutricionais: desafios para a área de Nutrição. CienSaudeColet 2013; 18(2):294-294.

3. Ministério da Saúde [homepage na internet]. Com obesidade em alta, pesquisa mostra brasileiros iniciando vida mais saudável [acesso em 30 jun 2019]. Disponível em:http://www.saude.gov.br/noticias/agenciasaude/43604-apesar-de-obesidade-em-alta-pesquisa-mostra-brasileirosmais-saudaveis

4. Gonçalves CV, Mendoza-Sassi RA, Cesar JA, Castro NB, Bortolomedi AP. Índice de massa corporal e ganho de peso gestacional como fatores preditores de complicações e do desfecho da gravidez. RevBrasGinecolObstet 2012; 34(7): 304-9.

5. Pinheiro ARO, Freitas SFT, Corso ACT. Uma abordagem epidemiológica da obesidade. RevNutr. 2004; 17(4): 523-33.

6. Nogueira AI, Carreiro MP. Obesidade e gravidez. RevMed Minas Gerais. 2013;23(1):88-98.

7. Seabra G, Padilha PC, Queiroz JA, Saunders C. Sobrepeso e obesidade pré-gestacionais: prevalência e desfechos associados à gestação, RevBrasGinecolObstet 2011; 33(11):348-353.

8. Santos KCR, Muraro LO, Witkowski MC, Breigeiron MK. Ganho de peso gestacional e estado nutricional do neonato: um estudo descritivo. Rev Gaúcha Enferm. 2014 mar; 35(1):62-69.

9. Melo ME. Ganho de peso na gestação. Associação Brasileira para o Estudo da Obesidade e da Síndrome Metabólica - ABESO, 2011.

10. Parizzi MR, Fonseca JGM. Nutrição na gravidez e na lactação. RevMed Minas Gerais 2010; 20(3):341-353.

11. Organização Pan-Americana da Saúde. M Sociedade Brasileira de Diabetes Rastreamento e diagnóstico de diabetes Mellitus gestacional no Brasil. Brasília: OPAS; 2017 [acesso 2019 jul 29] Disponível em:https://www. diabetes.org.br/profissionais/images/pdf/diabetes-gestacional-relatorio.pdf

12. Weinert LS, Silveiro SP, Oppermann ML, Salazar CC, Simionato BM, Siebeneichler A, et al. Diabetes gestacional: um algoritmo de tratamento multidisciplinar. ArqBrasEndocrinolMetab. 2011; 55(7):435-45.

13. Ministério da Saúde[homepage na internet]. Hipertensão (pressão alta): o que é, causas, sintomas, diagnóstico, tratamento e prevenção [acesso em 30 jun 2019]. Disponível em:http://www.saude.gov.br/saude-de-a-z/ hipertensao

14. Moura MDR, Castro MP, Margotto PR, Rugolo LMSS. Hipertensão arterial na gestação: importância do seguimento materno no desfecho neonatal. Comun. ciênc. saúde 2011;22: 113-120.

15. Moura ERF, Oliveira CGS, Damasceno AKC, Pereira MMQ. Fatores de risco para síndrome hipertensiva especifica da gestação entre mulheres hospitalizadas com pré-eclâmpsia. CogitareEnfermAbr/Jun 2010;15(2):250255.

16. Macedo LO, Monteiro DLM, Mendes BG. Obesidade e pré-eclampsia. FEMINA Mar/Abr 2015;43(2):83-88.

17. Kalil JÁ, Jovino MA, Lima MA, Kalil R, Magliari MER, Santo MKD. Investigação da trbose venosa profunda. J VascBras 2008; 7(1):28-37.
18. Secretaria da Saúde do Paraná [homepage na internet]. Conceitos e definições [acesso em 30 jun 2019]. Disponível em: http://www.saude. pr.gov.br/modules/conteudo/conteudo.php?conteudo $=668$

19. Aveiro NMP. A infertilidade na mulher obesa. Coimbra: Faculdade de Farmácia da Universidade de Coimbra,2014.

20. Amorim MMR, Leite DFB, Gadella TGN, Muniz AGV, Melo ASO, Rocha AM. Fatores de risco para macrossomia em recém-nascidos de uma maternidade-escola no Nordeste do Brasil. RevBrasGinecolObstet 2009; 31(5):241-248

21. Valle CP, Durce K, Ferreira CAS. Conseqüências fetais da obesidade gestacional. O mundo da Saúde, 2008 Out-Dez; 32(4):537-41.

22. Fiorelli LR, Zugaib M. Resultado perinatal na macrossomia fetal. RevMed (São Paulo). 2007 jul.-set.;86(3):144-7. 